\title{
Fred Wander et la censure
}

Alfred Prédhumeau, Fred Wander und die Zensur

Alfred Prédhumeau, Fred Wander and censorship

\section{Alfred Prédhumeau}

\section{OpenEdition}

\section{Journals}

Édition électronique

URL : https://journals.openedition.org/austriaca/884

DOI : 10.4000/austriaca.884

ISSN : 2729-0603

\section{Éditeur}

Presses universitaires de Rouen et du Havre

\section{Édition imprimée}

Date de publication : 1 décembre 2019

Pagination : 235-254

ISBN : 979-10-240-1454-8

ISSN : 0396-4590

\section{Référence électronique}

Alfred Prédhumeau, « Fred Wander et la censure », Austriaca [En ligne], 88-89 | 2019, mis en ligne le 31 décembre 2020, consulté le 24 octobre 2022. URL : http://journals.openedition.org/austriaca/884 DOI : https://doi.org/10.4000/austriaca.884 
Alfred PrédHumeau

Université Toulouse Jean-Jaurès,

Centre de recherches et d'études germaniques (CREG, EA 4151)

\section{Fred Wander et la censure}

\section{Introduction : repères théoriques}

S'agissant de production symbolique, la contrainte que le marché exerce par l'intermédiaire de l'anticipation de chances de profit prend naturellement la forme d'une censure anticipée, d'une autocensure, qui détermine non seulement la manière de dire $[\ldots]$ mais aussi ce qui pourra et ne pourra pas être dit ${ }^{1}$.

Pierre Bourdieu, dans ces quelques lignes, résume au mieux l'enjeu de toute politique de censure : assurer l'intériorisation consciente ou inconsciente de l'habitus qui permettra au producteur de langage, locuteur ou écrivain, d'avoir accès au marché et de lui procurer en retour un profit. Car il s'agit bel et bien de la définition d'une politique d'échange - échange qui dans l'esprit de Bourdieu n'est pas exclusivement économique, même si, dans le cas des écrivains, dépend de cette capacité d'échange économique - texte contre argent - la pure et simple condition sociale de pouvoir vivre de son métier. Bourdieu ajoutera plus loin :

La censure n'est jamais aussi parfaite et aussi invisible que lorsque chaque agent n’a rien à dire que ce qu'il est objectivement autorisé à dire : il n'a même pas à être, en ce cas, son propre censeur, puisqu'il est en quelque sorte une fois pour toutes censuré, à travers les formes de perception et d'expression qu'il a intériorisées et qui imposent leur forme à toutes ses expressions ${ }^{2}$.

Bourdieu introduit alors la fonction «d'agent», personne ou pouvoir habilité à exercer un contrôle sur les lieux de passage de cet échange entre le producteur textuel et le consommateur de ces textes - peu importe qu'il s'agisse de textes politiques, philosophiques, de littérature, etc. L'agent devient alors le seuil critique du processus de productions textuelles qui autorisera le producteur à avoir accès, ou non, au marché

1. Pierre Bourdieu, Langage et pouvoir symbolique, Paris, Seuil, 2001, p. 115.

2. Ibid., p. 345 . 
qui, en retour, lui accordera statut et/ou subsistance, voire fortune. La censure, sous toutes ses formes, est le maillon critique de la transmission entre les instances de la chaîne de production, dans le cas de Wander, littéraire en RDA. Nous allons tenter dans ce qui suit d'en donner quelques aperçus.

L'écrivain Fred Wander ${ }^{3}$, né en 1917 à Vienne, émigré à l'âge de 21 ans, supplicié des camps d'esclavage nazis, dépendances d'Auschwitz et Groß-Rosen, libéré de Buchenwald en 1945, adhérent au KPÖ à la fin des années 40 et délégué de ce parti à l'Institut de littérature à Leipzig, n'était probablement pas destiné, vu ses origines et sa formation modestes, à embrasser une carrière d'écrivain. D’ailleurs, après sa libération des camps, c'est en tant que reporter d'un quotidien communiste du soir, Der Abend, où Bruno Frei le remarqua et le délégua en 1955 comme "écrivain» autrichien aux premiers cours de l'Institut de littérature à Leipzig ${ }^{4}$, qu' il commença à vivre de ses écrits. Fred Wander n’avait jusqu'alors écrit que des reportages ou des nouvelles ${ }^{5}$, essentiellement pour la jeunesse, pour lesquelles il n'avait pas trouvé d'éditeur'.

3. Nous ne résumerons pas ici la biographie de Fred Wander, mais renvoyons au résumé que nous en donnons dans notre article «Fred Wander : un Schlemihl viennois en France», dans "Émigration et mythe. L'héritage culturel de l'espace germanique dans l'exil à l'époque du national-socialisme», Andrea Chartier Bunzel, Mechthild Coustillac et Yves Bizeul (dir.), Cahiers d'études germaniques, nº 76, 2019, p. 223-236. Ces indications étant elles-mêmes une version très abrégée de notre biographie littéraire de Fred Wander en préparation et dont la publication est prévue pour 2021.

4. Bruno Frei (1897-1988), né à Bratislava, fut journaliste à Berlin et émigra en 1933 à Prague puis en France où il fut interné au camp du Vernet d'Ariège. Il émigra ensuite au Mexique pour rentrer en 1947 à Vienne où il devint rédacteur en chef du Abend communiste. Voir la notice de Laure Gallouët dans Hélène Leclerc (dir.), Le Sud-Ouest de la France et les Pyrénées dans la mémoire des pays de langue allemande au XXe siècle, Toulouse, Le Pérégrinateur, 2018, p. 62-63.

5. Dernièrement fut trouvée dans les archives une publication sous pseudonyme probablement de la plume de Wander. Celle-ci, cependant, il est bon de le préciser, est de piètre qualité et relève de la catégorie germanophone des Groschenhefte, une littérature de kiosque qui n’a pas son pareil en France. Voir Marion Horn, «Roger Grillons Das Geheimnis der Rue de Bourgogne. Fred Wanders erste literarische Veröffentlichung im Jahr 1950?», Erzählen zum Überleben. Ein Fred Wander Handbuch, Walter Grünzweig et al. (éd.), Vienne, Theodor Kramer Gesellschaft, 2019, p. 226-235. Cette brochure sera rééditée en RDA en 1957, toujours sous pseudonyme, ce qui semble indiquer que l'auteur lui-même ne tenait pas ce texte en haute estime. Voir le dossier de censure aux Archives fédérales allemandes (AFA), DR 1/3987, autorisation d'imprimer $n^{\circ} 305 / 74 / 57$.

6. On ne sait rien de précis concernant ces refus de publication. Auraient-ils eu des raisons esthétiques ou idéologiques? Il est certain qu'en ces années d'après-guerre plusieurs facteurs de censure structurale étaient susceptibles d'empêcher une publication de certaines œuvres. 


\section{Précensure par formatage idéologique}

Bruno Frei délègue donc Wander à l'Institut de littérature qui, plus tard, sera nommé Johannes R. Becher. C’est pour le journaliste une opportunité sous plusieurs aspects : il échappe ainsi à son conflit amoureux entre sa femme Ottilie et Maxie Brunner, sa future deuxième épouse, il apprend en outre les bases du métier d'écrivain tout en profitant de cours de littérature ou de philosophie (marxiste) et il a enfin la possibilité de proposer son premier manuscrit à un éditeur de RDA qui va en accepter la publication. La fondation de cet institut avait été accompagnée des sarcasmes de l'écrivain qui lui prêterait bientôt son nom :

Der noch zu gründenden Akademie der Künste wird ein Entwurf zur Bildung eines Literatur-Erziehungs-Instituts (Internats) eingereicht, als Mittel, realistische Kunst zu erzielen. Im Kampf gegen den Formalismus hyperformalistischer Retortenexperimente. „Ideologische Durchblutung" wäre die Aufgabe des LiteraturSeminars, meint der unfreiwillige Spaßmacher ${ }^{7}$.

Le débat qui se cache derrière cette ironie acerbe n'est que de façade, car il ne s'agit pas de savoir si le talent s'apprend ou si on peut l'enseigner, mais de déceler les talents et de les former aux principes du «réalisme socialiste». Cette notion elle-même est bien entendu fluctuante dans sa signification et ses diverses applications, mais en résumé, comme l'a signalé l'ami de Wander, Erich Loest, il s'agissait de produire des écrivains de parti ${ }^{8}$. En fait, pour accéder à ce statut, il fallait déjà être un écrivain très proche du parti unique, même si, ironie du paradoxe, cet Institut produira de nombreux opposants au régime...

Ce qui ne manque pas de frapper, c'est que les directives littéraires émanant des enseignements officiels de l'Institut, dans la mesure où elles avaient pour but d'inciter les écrivains à se tourner de manière résolue et engagée vers la réalité supputée, c'est-à-dire en conformité avec la lecture qu'en donnaient les cadres du parti, restaient sans influence sur les sujets des œuvres de Wander : il semble être un écrivain qui vit en RDA, mais sur lequel la vie de ce pays n’a aucune prise littéraire. Cette année de formation à l'Institut semble au contraire l'avoir confirmé dans son

7. Johannes R. Becher dans son journal de 1950. Cité d’après : Isabelle Lehn et al., Schreiben lernen im Sozialismus. Das Institut für Literatur „Johannes R. Becher", Göttingen, Wallstein, 2018, p. 32. Le plaisantin involontaire, c'est Alfred Kurella, premier directeur de l'Institut et grand adversaire de Becher.

8. Ibid., p. 38. 
habitus, acquis dans les camps, qui consiste à faire profil bas ${ }^{9}$. Pour s'en rendre compte, il suffit de comparer ce qu'il dit dans ses mémoires de cette expérience à Leipzig et ce que l'on peut trouver comme informations le concernant dans l'ouvrage Schreiben lernen im Sozialismus ${ }^{10}$. Celui-ci rend largement compte du débat poétologique entre Wieland Herzfelde et Lukács, par Alfred Kurella interposé. Ce débat remontant au congrès d'écrivains de Moscou en 1934, où Radek avait sonné la charge contre la littérature moderne des Joyce et autres «décadents bourgeois », et qui avait été théorisé par Lukács dans son essai Erzählen oder Beschreiben ${ }^{11}$, avait fait l'objet d'un séminaire de Herzfelde à Leipzig $^{12}$. Si les comptes rendus sont exacts, Wander ne participa pas à cette controverse. Il en va de même pour la participation des étudiants de l'Institut au IVe Congrès des écrivains de RDA qui se tint à Berlin, en janvier 1956. Anna Seghers y avait tenté une timide critique de la littérature de $\mathrm{RDA}$, procédant, d'après elle, de manière trop schématique, et, avec Arnold Zweig, en avait appelé au courage de critiquer certaines malformations de la société socialiste ${ }^{13}$. La réaction de l'appareil contredisant cette affirmation ne se fit pas attendre : il n'est pas besoin de courage pour critiquer - sous-entendu : qu'y a-t-il à critiquer ? L'étudiant Ralph Giordano ${ }^{14}$ monta à la tribune pour interpeller le conformisme de ce qu'il appela «la dictature du goût petit-bourgeois au nom du prolétariat ${ }^{15}$ ». Wander ne se manifesta pas par rapport à cette discus-

9. Fred Wander, Das gute Leben oder von der Fröhlichkeit im Schrecken. Erinnerungen [Leben], Göttingen, Wallstein, $2^{e}$ édition, 2006, p. 153-177.

10. Voir Schreiben lernen, op. cit., dont le chapitre concernant le premier cours prend souvent Leben à témoin, ce qui, à notre avis, doit être fait avec beaucoup de perspicacité, la première rédaction de cet ouvrage remontant aux années 90 , donc avec un décalage de près d'un demi-siècle.

11. Georg Lukács, Erzählen oder beschreiben. Zur Diskussion über Naturalismus und Formalismus [1936], dans Werke, Probleme des Realismus I, vol. 4, Neuwied, Luchterhand, 1971, p. 197-242.

12. Les séminaires de Herzfelde se passaient l'observation du directeur Kurella. Il en existe des comptes rendus relativement précis. Voir Schreiben lernen (op. cit., p. 155 et suiv.), qui paraphrase les contenus de l'exposé de Herzfelde et des débats.

13. Bourdieu consacre un chapitre tout à fait pertinent dans ce contexte, intitulé : «Décrire et prescrire : les conditions de possibilité et les limites de l'efficacité politique» (Langage et pouvoir symbolique, op. cit., p. 187-198). Décrire et prescrire semblent être la vérité cachée de Erzählen oder Beschreiben (op. cit.)!

14. Ralph Giordano (1923-2014), écrivain et journaliste, est connu essentiellement pour son roman Die Bertinis. Il avait été adhérent du KPD clandestin en RFA mais rompit avec le communisme après son séjour à l'Institut. Il en rendit compte dans son ouvrage Die Partei hat immer recht.

15. Schreiben lernen, op. cit., p. 167-168 : «die Diktatur des kleinbürgerlichen Geschmacks 
sion. Il en fut de même lors des discussions internes faisant suite au $\mathrm{XX}^{\mathrm{e}}$ Congrès du Parti communiste d'Union soviétique et du rapport Khrouchtchev. Si l'écrivain exprime son admiration pour Loes ${ }^{16}$ et Giordano, il constate pour lui-même qu'il fut incapable de participer à cet engagement :

Und wenn ich heute, aus der Entfernung der vielen Jahre, die tiefe Bewegung bei Giordano, Loest und vielen anderen sehe, die Erschütterung, welche diese Ereignisse bei ihnen ausgelöst hatten, dann frage ich mich beunruhigt - woran liegt es, dass alle diese Tatsachen an dir vorbeigerauscht sind und du auch nie eine Zeile darüber geschrieben hast ${ }^{17}$.

Wander tente néanmoins de donner une réponse à son silence : «[M]eine tief verwurzelte Vorsicht, die man in totalitären Lagern zum Überleben brauchte, hatte noch Wirkung über mich!» Il y aurait donc en RDA un effet d'autocensure causé par l'expérience nazie. Ce qui revient à penser que, pour Wander, il existe à tout le moins une similitude entre les deux régimes, débouchant chez lui sur une censure structurale. Il est vrai qu'il n’a rédigé cette explication que bien plus tard, après la chute des régimes communistes, alors que, très longtemps, il resta adhérent du KPÖ et ne trouva, officiellement, rien à redire au régime de RDA. D'ailleurs, lorsqu'il affirme qu'il n'était pas un écrivain militant - «ein militanter Bücherschreiber war ich nie ${ }^{18} »-$ on peut ajouter que le reporter, lui, fut militant, car ses sujets étaient toujours proches des soucis des milieux populaires viennois. Ne se pourrait-il pas, et cela se comprendrait tout à fait, qu'il n'ait pas voulu être renvoyé de l'Institut, ce qui aurait mis un terme à sa délégation ? En effet, pour pouvoir séjourner en RDA, il lui fallait l'aval de son parti, et il ne souhaitait pas rentrer à Vienne.

im Namen des Proletariats». À l'Institut, cet incident donna lieu à une réunion de debriefing avec le directeur Kurella.

16. Erich Loest (1926-2013), écrivain et journaliste, avait adhéré au SED en 1947. Il en fut exclu en 1957 et condamné à un emprisonnement de plus de sept ans à Bautzen. Il émigra en 1981 en RFA, revint à Leipzig après la disparition de celle-ci. Plusieurs de ses œuvres relatent sa persécution par la dictature poststalinienne est-allemande, en particulier Durch die Erde ein Riss (1981).

17. Das gute Leben, op. cit., p. 170. L'attitude de son épouse Maxie sera à l'opposé de la sienne : elle s'impliquera sans relâche dans les problèmes de la vie quotidienne des femmes en RDA.

18. Ibid., p. 171. 


\section{Censure viennoise}

Depuis son retour en Autriche, l'auteur projetait d'écrire un roman qu'il voulait intituler Hekuba:

[I]ch hatte im Kopf eine Geschichte, die ich schreiben wollte: Ein Junge kommt aus dem Krieg zurück und sucht den Mörder seines Vaters! Der Vater, ein Widerstandskämpfer, war von der GESTAPO entdeckt, verhaftet und hingerichtet worden. Der Verräter war der Bruder des Vaters. Die Mutter lebte nun mit dem Verräter. Hamlet! [...] Und es schien fast, wenn ich durch die Straßen lief, als suchte ich den Mörder meines eigenen Vaters ${ }^{19}$.

À l'Institut de Leipzig, il pensait qu'il aurait le temps de continuer son travail sur cette œuvre qui lui tenait particulièrement à cœur : «Ich hatte die Arbeit daran lange Zeit unterbrochen. Nun knöpfte ich mir das Manuskript wieder vor ${ }^{20}$.» Cependant, ce roman ne vit jamais le jour. Que s'était-il passé ? Dans ses mémoires, il résume la situation ainsi :

Das war jener Roman, der sich wie ein Ballon blähte und bald vierhundert Seiten hat. Martin, der Held meiner Geschichte, verfolgt den Mörder seines Vaters, doch als er ihm dann gegenübersteht und seine heuchlerische Fratze sieht - lässt er die Waffe sinken! Persönliche Rache ist sinnlos geworden ${ }^{21}$.

Wander insinue qu'il s'était rendu compte que la complexité du sujet lui en rendait la rédaction impossible. Il lui fallait donc trouver une intrigue moins ambitieuse. Phénomène d'autocensure pour prise de conscience de la limite de ses compétences littéraires ? Il se serait rendu compte, au contact d'un texte de Camus, "La crise de l'homme», dont il avait pris connaissance par une traduction allemande abrégée, publiée par la presse américaine d'occupation en Autriche, Die Amerikanische Rundschau, que l'auteur français aurait atteint les limites de sa capacité de compréhension des phénomènes criminels nazis à cause de son appartenance de classe à la couche «des intellectuels bourgeois». De là Wander développa une esquisse concernant la fonction de l'intellectuel contre les manifestations "d'américanisme» et, en corollaire, de rejet d'humanité comme manifestation de recherche de pouvoir total, voire totalitaire. Il en a développé l'idée dans un exposé de ce roman retrouvé dans les archives :

19. Ibid., p. 118.

20. Ibid., p. 162.

21. Ibid., p. 162. 
Es ist ihre [der Schriftsteller] heilige Pflicht das Gift zu entfernen und die Menschen zu lehren, ihre Mitbürger nicht nach dem Erfolg, sondern allein nach dem Begriff der Würde zu beurteilen. Es ist ihre Pflicht den Menschen wieder Werte zu geben, die ewigen Werte der Humanität. Natürlich müssen wir beginnen, das Gift aus unseren eigenen Herzen zu entfernen. Aber das allein genügt nicht. Es gilt zu handeln und einen geschlossenen und unerbittlichen Kampf zu führen, gegen jene, die der Welt einen neuen heraufkommenden Terror bescheren wollen ${ }^{22}$.

En fait, il semble que ce projet de proposer une version "prolétarienne» et romancée du diagnostic de Camus ait été un échec. C’est du moins l'interprétation qu'en donne Karl Müller dans un article récent, après avoir résumé l'exposé que rédigea Wander entre 1958 et $1960^{23}$. Le lectorat du communiste Globus Verlag de Vienne, après avoir demandé de multiples corrections et tergiversé, lui annonça en avril 1960 qu'il renonçait à la publication, car c'était devenu un Kolportage-Roman, de la littérature de colporteur - un argument peu élogieux. Comme Wander a, semble-t-il, voulu effacer toute trace de cette humiliation, aucun reste de manuscrit n'a pu être découvert, ni à Vienne ni au Mitteldeutscher Verlag à Halle, avec lequel le projet avait été aussi négocié, probablement comme coédition.

$\mathrm{Si}$, cependant, les conjectures de Müller sont exactes, nous sommes face à un exemple quasiment prototypique de "censure structurale» telle que Bourdieu l'a définie : le lecteur de l'éditeur a poussé, probablement de manière inconsciente, l'auteur dans une direction l'éloignant de plus en plus de son intention première, romancer les thèses de Camus, pour le pousser sur une voie communiste orthodoxe de "prolétaires» en lutte, jusqu'à constater finalement que la qualité littéraire n’y était pas. Le lecteur ayant intégré de manière «totale» les critères du réalisme socialiste, il finit par empêcher la publication d'un roman ne correspondant pas à ces critères, le «sur-moi» du parti ayant commandé à la décision finale. D'ailleurs, le sur-moi du parti pouvait tout aussi bien être la précensure de RDA aux étages multiples. Dans ce cas, celle-ci, agissant dans l'ombre, pouvait conseiller à distance, oralement, le lecteur viennois de l'appréciation orthodoxe de ce manuscrit. Comme la RDA était le financier des organisations et structures communistes autant en Autriche qu'en RFA, il était évidemment bon de suivre les «conseils» si généreusement distillés.

22. Wander, exposé pour Hekuba, p. 15, cité d’après Karl Müller, « „Die Zeit ist aus den Fugen.“ Fred Wanders Hekuba-Projekt - ein Wiener Roman. Zur Camus- und Shakespearerezeption in der Nachkriegszeit", dans Erzählen zum Überleben, op. cit., p. 60.

23. Karl Müller, art. cité, p. 52-67. 


\section{Wander et la censure en RDA}

Nonobstant sa tiédeur face aux idéologies orthodoxes, Wander restera de très longues années un auteur apprécié de l'édition estallemande : livres de jeunesse, livres de voyages, livres mémoriels de la Shoah et de l'exil qui lui procurent de forts tirages et des revenus réguliers. Cette diversité de l'œuvre rend une classification littéraire de l'auteur complexe : est-il un auteur autrichien, définition qu'il aurait refusée en ces années avec indignation, fait-il partie de la littérature de l'exil, à titre rétroactif pour ainsi dire, est-il un auteur de RDA, alors qu'il ne participe à aucun des grands débats qui agitent régulièrement la scène littéraire de ce pays ? Ajoutons ici, sans vouloir insister, que la tiédeur idéologique inclut une participation active, d'abord aux services d'espionnage militaire de RDA où il fut enrôlé pendant de brèves années afin de collationner des informations en France, puis par la firme horch-und-guck (bouclier et glaive du parti), plus connue sous le nom de Stasi. Il y fut longtemps «collaborateur informel», IM. S'il ne semble jamais avoir accepté cette collaboration avec zèle, ni d'avoir été d'une très grande utilité pour les services concernés, il n'en reste pas moins que, vu ses contacts multiples avec les milieux littéraires berlinois, incluant par exemple le couple Wolf, il était susceptible de communiquer des informations qui, recoupées avec d'autres, permettaient de se faire une idée de la fiabilité et la fidélité à la cause communiste de ces écrivains-cibles.

Nous ne passerons pas en revue l'ensemble des œuvres soumises à la précensure $^{24}$ et publiées pendant ces années-là, mais nous concentrerons sur quelques exemples qui nous paraissent typiques.

Nicole, un cas de «censure positive»?

Le cas que nous annonçons en titre de ce paragraphe n'est pas prévu, nous semble-t-il, par les théories concernant la «censure structurale»:

24. Je ne peux, dans le cadre de cet article, exposer les rouages de la censure en RDA. Pour une première information, je renvoie à ma thèse, Alfred Döblin, November 1918, révolution et mystique, où j'expose, p. 149-151, le système mis en place auprès du ministère de la Culture, celle, décisive finalement, auprès du secrétaire du Bureau politique du SED chargé des questions idéologiques, Kurt Hager, dirigé par la redoutable Ursula Ragwitz restant ici dans l'ombre. Pour de plus amples informations, on se reportera à Robert Darnton, De la censure, J.-F. Sené (trad.), Paris, Gallimard, 2014, p. 179-288. 
il s'agit d'une situation où un ouvrage est publié malgré sa faiblesse ostensible en termes de qualité littéraire. La précensure lui trouve donc des qualités autres que celles que l'on est en droit d'attendre d'une œuvre littéraire. À notre avis, cela vaut pour Nicole $e^{25}$.

Ce court roman paraît en 1971 dans une collection pour livres de jeunesse. Vu la thématique, il s'agit d'un Bildungsroman, du roman de formation d'une jeune femme originaire de Grenade, non loin de Toulouse, qui «monte» à Paris pour y faire sa vie ${ }^{26}$. L'intrigue est située au début des années soixante, la guerre d'Algérie et le terrorisme de l'OAS sont alors à leur paroxysme et, malgré la croissance des «trente glorieuses», les luttes sociales sont à l'ordre du jour. Wander a imaginé des coulisses socio-culturelles qui sont une anticipation de la période post-soixante-huit. L'auteur crée des personnages qui sortent droit d'un moule, il en crée une multitude, et ils ne sont donc travaillés ni dans leurs profils sociaux - les clichés prédominent - et encore moins dans leurs psychologies, ce qui a pour résultat que leurs actions ou interactions paraissent immotivées ${ }^{27}$. L'avis qualitatif des censeurs est plutôt critique et le service chargé de ce travail en utilisa quatre, alors que pour ce genre de texte un censeur, au maximum deux eussent dû suffire. Ce qui montre bien que l'œuvre posait problème. Tout donne à penser que Nicole était au départ destiné à un public adulte : Wander s'est plaint que la censure lui avait rayé les passages érotiques ${ }^{28}$, la sexualité n'étant pas inscrite au programme de Bitterfeld, ni des Bildungsromane de l'ère Ulbricht déclinante, pudibonderie qui avait prévalu en RFA aussi jusqu'au milieu des années soixante ${ }^{29}$.

25. Fred Wander, Nicole, Neues Leben, 1971.

26. Rappelons que cela faisait partie des tâches du «collaborateur» Wander que de fournir des informations sur l'OAS et le PCF. On pourrait donc, si on était malveillant, considérer ce petit livre comme un travail de "commande».

27. Il faut signaler que l'expert de l'œuvre de Wander, Walter Grünzweig, auteur d'un article concernant ce roman, est d'un avis contraire : selon lui, dans ce texte, y compris pour ce qui est de la qualité de production technique et des illustrations, tout est bon. Voir Walter Grünzweig, "Sinnbilder des Lebens. Fred Wander als Jugendbuchautor», dans Walter Grünzweig et Ursula Seebel (dir.), Fred Wander. Leben und Werk, Bonn, Weidle, 2005, p. 165-170. Notre opinion négative mérite donc d'être confrontée à un avis favorable et à la lecture de ce roman afin que le lecteur s'en fasse une idée personnelle.

28. Archives Fred Wander (AFW), 476, Archiv Preußischer Kulturbesitz, lettre à ses amis Draer du 29 décembre 1969.

29. Rappelons, à titre de comparaison, le slogan d'Adolphe Süsterhenn, politicien chrétien démocrate, prônant un «cinéma propre» par son Aktion saubere Leinwand en 1961. 
La conclusion d'un des censeurs est caractéristique de l'ensemble des «expertises», y compris celle de l'éditeur, supposé pourtant défendre le manuscrit qu'il souhaite voir publié :

Enttäuscht wird man nur sein, wenn man eine geschlossene Erzählung erwartet: denn für ein solches literarisches Genre fehlt die interne Verbindung von Privatleben und Öffentlichkeit; in vieler Beziehung besteht diese Verbindung nur äußerlich. Das endet dann direkt in plakativen Zeilen über Politik ${ }^{30}$.

Pourquoi donc avoir publié ce texte ? Car, en outre, un autre censeur constate :

Diffizil ist auch die Darstellung der Kommunistischen Partei oder ihrer Rolle bei der Führung der Massen. Es ist selbstverständlich nicht einfach, dieses Thema in einem so kleinen Buch exakt zu behandeln, und so kommt es dann dazu, daß Fred Wander die Kommunisten kaum in Aktion zeigt ${ }^{31}$.

On peut simplement supposer que Wander, pour cette publication, a joui d'une conjoncture très favorable, due éventuellement à des soutiens externes à l'édition. Sa carrière en RDA était sur une bonne voie.

\section{La censure face à un chef d'œuvre}

En 1971 parait Der siebente Brunnen, l'œuvre sans nul doute la plus connue de Fred Wander ${ }^{32}$. C'est une construction littéraire hardie où l'auteur tente de maitriser l'horreur concentrationnaire, à la fois à l'aide de moyens stylistiques réalistes et de fictionnalisations osées, ces dernières ayant aussi pour fonction de documenter la pérennité de la culture yiddish qui survit à l'aide de la narration (Erzählung), d'origine orale, mais fixée grâce à une tradition scripturale qui réinterprète et réécrit cette fiction afin que la réalité de la Shoah soit transmise de génération en génération ${ }^{33}$. Wander est un acteur-narrateur de cette chaîne transgénérationnelle germanophone.

30. AFA, DR 1/ 3544a, p. 405, souligné par le censeur.

31. Ibid., p. 396.

32. Die siebente Brunnen. Erzählung, Berlin-Weimar, Aufbau, 1971.

33. On consultera à ce sujet le très bel article-hommage de Martine Benoit, «Chelm liegt nicht mehr auf dieser Welt. Die Erinnerung an das Ostjudentum in Fred Wanders Der siebente Brunnen», dans Erzählen zum Überleben, op. cit., p. 98-103. Elle y consacre quelques lignes fort instructives à l'utilisation du yiddish comme moyen de distanciation entre la narration de Wander et le lecteur allemand, afin d'empêcher ce dernier de se parer d'empathie (p. 100). C'est une idée intéressante qui signifierait que l'on pourrait 
De par sa mouture exceptionnelle, le texte pose, a priori, un énorme problème idéologique : la résistance active n'y est guère mise en valeur, ce qui prévaut étant la souffrance, l'absence d'espoir ou d'issue positive, il va donc à l'encontre de la voie héroïque dictée à Bitterfeld. Même les passages se rapportant à Buchenwald ne font pas référence aux organisations clandestines communistes, mais se passent aux baraquements de quarantaine, dans une rare fusion entre le genre littéraire carcéral et celui de la quarantaine «hospitalière » - genres dont nous savons, depuis Dostoïevski, qu'ils ne sont pas que la transcription réaliste, mimétique, d'un monde cruel dont on se débarrassera à l'aide de la Révolution socialiste, comme le veut le message de Bruno Apitz, après intervention de la censure ${ }^{34}$, mais la métaphore d'un monde abandonné par Dieu où les forces du mal semblent avoir définitivement pris le pouvoir - politique, philosophique et religieux.

À l'opposé de ce qui s'était passé pour Bruno Apitz, la censure, du moins dans les parties que nous pouvons relire et contrôler, n'y a pas trouvé à redire. Pour bien montrer que le régime était favorable à la diffusion et à la lecture de cette œuvre, Wander obtint, en 1972, le prestigieux Heinrich-Mann-Preis, consécration littéraire définitive de tout auteur en RDA. Certaines pages de ces documents de censure sont des hommages appuyés à la qualité littéraire de cette œuvre, bien que Wander n'ait pas écrit et consacré Der siebente Brunnen à la gloire de la résistance organisée :

Doch um keine Zweifel aufkommen zu lassen: die Arbeit ist nicht dem organisierten antifaschistischen Widerstand gewidmet, obwohl er an einigen Stellen spürbar wird. Sie berührt Bereiche, in die er kaum gelangte, kaum gelangen konnte. Des Autors Sphäre ist der Bodensatz der Lager, aus ihm lässt er seine Gestalten entstehen.

traduire ces passages en français, car nos lecteurs francophones auraient droit à l'empathie. Nous ne partageons pas cet avis : le lecteur francophone a probablement lui aussi, même si c'est à un degré différent, besoin, non pas d'interdit d'empathie, mais d'interdit d'empathie «bon marché». Il s'agit, pensons-nous, de prendre conscience de la singularité des crimes perpétrés par les nazis, avec la complicité de la majorité des populations germanophones et de leurs alliés à ambitions "sol et sang aryens", dont, malheureusement, une partie non négligeable d'acteurs français qui, grâce à la complicité suisse (francophone), livrèrent Wander aux mains des nazis pour esclavage et, probablement, mort assurée.

34. Bruno Apitz, Nackt unter Wölfen, Halle, Mitteldeutscher Verlag, 1958. Cette version est une version fortement retravaillée avec l'aide de différentes instances de censure structurale est-allemande. Voir Susanne Hantke, Schreiben und Tilgen. Bruno Apitz und die Entstehung des Buchenwald-Romans "Nackt unter Wölfen", Fondation du Mémorial Buchenwald et Mittelbau-Dora, Göttingen, Wallenstein, 2018. 
Die so tief Gedemütigten und Erniedrigten, die der Vernichtung Bestimmten, die kaum noch einer Rettung Zugänglichen, treten unter der Feder des Autors hervor, gewinnen Schönheit und vergegenwärtigen, welcher Reichtum an Menschlichkeit durch die Nazibarbarei verloren gegangen ist. [...] Die meisten gehen unter. Unter schrecklichen Bedingungen. So war es ${ }^{35}$.

\section{Et le second censeur remarque :}

Unerträglich wäre die Summe der Widerstandslosigkeit, die hier schonungslos an den Tag gebracht wird, stände nicht der Ich-Erzähler hinter allem mit ständigem Forschen eben nach dem Widerstand. Es gibt unter diesen Häftlingen kein Anzeichen für eine Selbstorganisation zur Abwehr gegen die Peiniger. Nicht einmal in Buchenwald werden diese Spätkommenden in ihren Quarantäneblocks von der Organisation der antifaschistischen Kämpfer erfasst. [A]ll das wäre in der Summe von Fatalismus unerträglich, hätte nicht der Autor in einigen wenigen Gestalten - einem Pariser Maquisard, einem sowjetischen Kriegsgefangenen, einem noch unterm Galgen mannhaften Antifaschisten - Gegenpole geschaffen $z u$ der geschilderten jüdischen Ausweglosigkeit ${ }^{36}$.

Ce censeur oublie toutefois de mentionner que l'action positive des antifascistes dans les camps est tout aussi désespérée que le fatalisme de certaines victimes juives (la grande majorité des susnommés périra), mais il s'agit de faire entrer le texte dans le moule de la doxa de l'héroïque antifascisme prolétarien. Wander ne manque cependant pas de souligner la courageuse intransigeance des suppliciés français : ce sont eux qui portent en eux le germe des victoires futures (et non le soldat de l'Armée rouge !). Pour exemple Pépé, militant communiste qui, dans Der siebente Brunnen, fait l'apologie de la révolution antinazie : «[...] gute Menschen können wir jetzt nicht brauchen, wir brauchen Recken, Kämpfer, Kopfabschlager, Messerschleifer,

35. AFA, DR1/2099a, p. 165-166.

36. Ibid., p. 170. Cette expertise est de la plume la plus redoutée, car dogmatique et intransigeante, des censeurs du ministère de la Culture, celle de $\mathrm{M}^{\mathrm{me}}$ Gärtner Scholle - nous n'insisterons pas sur le reproche d'origine antisémite de la jüdische Ausweglosigkeit. Pour cette censeure, voir dans la Berliner Zeitung du 9 mai 2008 l'article intitulé «Die Zensorin» de Siegfried Lokatis, un expert en histoire de l'édition, qui lui rend l'hommage suivant : "Ein wahrer Schrecken der schreibenden Männerwelt war allerdings Carola Gärtner-Scholle, die dienstälteste Gutachterin der Hauptverwaltung, die wegen ihres überschäumenden Temperaments keine verantwortliche Position bekleidete, sondern als eine Art Geheimrätin für Literatur im Verborgenen waltete.» Voir aussi Simone Barck et Siegfried Lokatis, Zensurspiele. Heimliche Literaturgeschichten aus der DDR, Halle, Mitteldeutscher Verlag, 2008. Ajoutons que cette censeure fut une résistante contre le régime nazi ; arrêtée en 1934, elle fut condamnée à trois années de forteresse. 
Ausbrecher. / Ihr werdet alle Sorten von Leuten brauchen, sagte ich [le narrateur] wenn die Revolution gemacht ist ${ }^{37}$."

De façon quelque peu inquiète, cette censeure mentionne le seul argument qui pourrait plaider contre une publication de cette œuvre : "Es erscheint reichlich spät und in einem sozialpolitischen Augenblick, wo die Gefahr besteht, dass die Darstellung dieser jüdischen Massenopfer die Sympathien der Leser in den falschen Kanal, nämlich in Sympathien für das heutige Israel lenken könnte ${ }^{38}$.» Et les amalgames de la tradition judéophobe ne se font pas attendre:

Doch darf man wohl annehmen, dass in unserer DDR die meisten Leser zu unterscheiden wissen, wer die wirklichen Erben der Hingemordeten sind, jene Israeliten, die das von der Walstreeet [sic] geschaffene und ausgehaltene Staatswesen als imperialistische Plattform unterstützen, die sich von den Erben der Mörder [la RFA] Gutmachung berappen lassen, oder jene junge Israeliten, die bei der Arbeit in ihren Genossenschaften gelernt haben, wo der Feind steht ${ }^{39} \ldots$

Cette censeure aussi plaide en faveur d'une publication, mais souhaite l'ajout d'une postface qui expliquerait aux lecteurs la différence entre les bons et les méchants Israéliens. Postface qui aurait à éviter le style de la méthode Holzhammer, le bourrage de crâne par la langue de bois. Sa suggestion de "censure constructive» échouera, Der siebente Brunnen sera bel et bien publié sans postface.

Pendant longtemps, Wander cherchera à publier Der siebente Brunnen en RFA. Si une première édition put finalement se faire en 1973 à Francfort-sur-le-Main au Röderberg-Verlag, éditeur très proche du Parti communiste, elle passa cependant inaperçue et ne contribua que très modestement à remplir de devises les caisses de RDA et de l'auteur. En 1985 parut enfin une édition chez Luchterhand qui aurait pu, sinon dû, signifier la percée de Wander en RFA. Il n'en fut rien toutefois, ce dont il se plaignit :

Mein "Brunnen“ hat übrigens in der BRD genau den Misserfolg, den alle vorausgesagt haben. Ich war vor wenigen Tagen bei Dr. Altenhein ${ }^{40}$ und er meint,

37. Der siebente Brunnen, op. cit., p. 84.

38. AFA, DR 1/2099a, p. 171.

39. Ibid.

40. Hans Altenhein fut directeur de Luchterhand de 1973 à 1987. Il fut un des acteurs principaux des échanges littéraires germano-allemands, tant et si bien que l'on nomma cette maison VEB Luchterhand. 
es wäre „Berührungsangst", warum die Kritiker, auf die es ankäme, das Buch nicht besprechen. (Es gab bisher eine Kritik in der FAZ, aber ein vernichtender Verriss, jawohl, meine Liebe $)^{41}$.

Ici les agents potentiels de transmission, les critiques de presse, semblent avoir préféré ne pas parler de cette œuvre dérangeante qui met le doigt littéraire dans la plaie béante des criminels nazis et de leurs complices. Pourquoi ? Seule une étude historique et socio-culturelle sur la lecture de la Shoah telle qu'elle est proposée dans les pays germanophones pourrait permettre de répondre à cette question. Nous ne nous voyons pas en mesure de pouvoir y répondre nous-même ici.

\section{Brunnen au cinéma?}

Le 29 mars 1983, la DEFA ${ }^{42}$ informa le scénariste Eberhard Görner que son scénario d'adaptation de Der siebente Brunnen soumis en 1982 avait été accepté et qu'il pouvait se mettre à la recherche d’un régisseur en respectant les adaptations de contenu discutées le 21 mars. Görner en trouva un en la personne d'Alexander Lang ${ }^{43}$.

Au préalable, le scénariste avait fait du lobbying lors du IV Congrès de l'Union des créateurs de cinéma et télévision de $\mathrm{RDA}^{44}$ et réclamé une décision en écrivant directement au Comité central du Parti socialiste unifié, le SED. Tout semblait donc mis sur d'excellents rails. Les cinéphiles experts en cinéma de RDA savent cependant que ce film n’apparut jamais sur les écrans, ni en RDA ni ailleurs, pas même parce qu'il fut interdit de représentation, mais simplement parce qu'il ne fut jamais tourné.

En fait, l'alarme négative était déjà incluse dans les impératifs consignés lors de la réunion d'accord :

$[M]$ it dem Szenaristen Görner gilt als fest verabredet, dass im Sinne der Balance von Nationalem und Sozialem jüdisch Rituelles nicht überzubetonen und niemals im Film ins Folkloristische auszuweiten ist, denn die Leidens- und

41. Lettre de Wander au Aufbau Verlag du 18 août 1985 (Archives de cet éditeur, Bibliothèque d'État, Berlin, IIIa 382607 0018). Il ne nous a malheureusement pas été possible de retrouver cette recension. Il en existe de plus récentes de la Frakfurter Allgemeine Zeitung qui cependant concernent des rééditions ultérieures. Quant à Die Zeit, Süddeutsche ou Spiegel, ces organes de presse semblent n'avoir rien publié en 1985.

42. Deutsche Film AG, société de production cinématographique de RDA qui exista de 1945, comme successeur de l'UFA, jusqu'à la fin de la RDA pour être vendue, après diverses transformations, à la société française Compagnie générale des eaux.

43. Fred Wander. Leben und Werk, op. cit., p. 55.

44. Verband der Film- und Fernsehschaffenden der DDR. 
Widerstandsfähigkeit jüdischer Menschen ist Beispiel, nicht Verabsolutierung (siehe Jacques, siehe Petrow $)^{45}$.

Avec cet argument, nous retournons donc aux sources de la quasi-interdiction du texte de Wander : la condamnation de la soi-disant passivité juive opposée à la résistance active des communistes - ici français et de la contribution décisive de l'Armée rouge à la défaite du Reich nazi. En fait, on est ici confronté à l'antisémitisme résiduel allemand se camouflant derrière une hérö̈sation des quelques acteurs capables d'opposition violente. Il y a, dans cette logique, de bonnes victimes et de moins bonnes.

En août 1983, le scénariste, qui ne souhaitait pas abandonner son projet, écrivit au «camarade» Kurt Hager pour en appeler à son antifascisme. Sur ce, Görner fut invité (convoqué ?) dans les bureaux de l'administration centrale «Cinéma» du ministère de la Culture en présence d'un responsable de la commission culture du Comité central du SED, délégué par Hager, afin, probablement, qu’on lui mît les points sur les «i». Le scénariste communiqua ceux-ci à Fred Wander peu après. Nous en résumons l'essentiel :

Les moments de passivité et de résignation dominent ; l'accumulation de violences face à la passivité n'est pas digne d'être portée à l'écran ; on cherche en vain, dans ce scénario, l'activisme politique des héros; il y en a eu à Buchenwald, le film veut raconter cette histoire de manière unilatérale ; on aurait pu en faire un film comme Nackt unter Wölfen, mais un film comme Brunnen n'est pas possible ${ }^{46}$.

Finalement, l'argument létal contre le tournage est le soupçon qui pèse sur les réalisateurs de vouloir produire un film d’art : «Der Stoff Der siebente Brunnen bedient nicht das Konzept der Massenwirksamkeit ; der bittere Ernst der Geschichte, die Authentizität, die niederdrückt, würde von Publikum sicher nicht angenommen ${ }^{47}$.» La référence citée comme preuve de cet avis est «l'échec» de Dein unbekannter Bruder, film d'Ulrich Weiß datant de 1982. En fait, c'est sur intervention du Bureau politique du SED, en la personne de Hermann Axen ${ }^{48}$, que ce film fut retiré des cinémas et du Festival de Cannes.

45. Fred Wander. Leben und Werk, op. cit., p. 54.

46. Ibid., p. 58.

47. Ibid., p. 59.

48. Hermann Axen (1916-1992), résistant, avait émigré en France et été interné au Vernet. Il était membre du Bureau politique du SED, depuis 1970 chargé des relations internationales. 
En 1990, après la chute du mur et peu avant la fin «officielle» de la $\mathrm{RDA}$, le scénariste tenta sa chance auprès de la $2^{\mathrm{e}}$ chaîne de télévision publique allemande, $Z D F$, et de la télévision publique bavaroise, la réponse de la seconde ressemblant étrangement à l'argument de «l'ennemi de classe » d'hier :

Ein sehr bewegendes Buch, eine sicherlich ungewöhnliche Art mit dem Thema KZ umzugehen. I Allerdings ein Thema, das es schwer und es in unserer „Kinolandschaft" zunehmend schwerer haben wird ${ }^{49}$.

Les lois implacables du marché remplacent celles du Bureau politique. Le grand public, intéressé ou non, ne verra donc jamais ce film ${ }^{50}$.

\section{Wander, censeur?}

En 2001 paraît une biographie de Maxie Wander qui était décédée en 1977 et dont les quelques œuvres publiées de son vivant et à titre posthume avaient connu un succès éditorial que l'on peut qualifier sans exagération aucune de phénoménal ${ }^{51}$. La biographie écrite par Sabine Zurmühl a la particularité de pouvoir s'appuyer sur une lecture non filtrée des journaux intimes de Maxie Wander, car si ceux-ci ont été publiés partiellement après le décès de Maxie, ils sont le résultat d'un travail de rédaction commun entre Fred Wander, l'éditeur et les instances de censure. Reprenons donc cette histoire dans un ordre chronologique.

L'épouse de Wander décède fin novembre 1977, début décembre l'écrivain parvient à écrire deux lettres à ses amis français où il fait état de son état de dévastation et des mesures qu'il prend pour tenter de

49. Fred Wander. Leben und Werk, op. cit., p. 65.

50. Ce paragraphe est basé sur l'article de Eberhard Görner, «Der siebente Brunnen von Fred Wander. Die Geschichte eines Films, der nicht gedreht wurde», dans Fred Wander. Leben und Werk, op. cit., p. 47-69.

51. Les journaux intimes de Maxie Wander seront publiés aussi bien en RDA qu'en RFA. D’abord au Buchverlag der Morgen en 1979 (Tagebücher und Briefe, Fred Wander éd.), puis en RFA (Leben wär' eine prima Alternative: Tagebuchaufzeichnungen und Briefe, Fred Wander éd., Neuwied, Luchterhand, 1980). Plus tard paraîtra Ein Leben ist nicht genug, Fred Wander (éd.), Neuwied, Luchterhand, 1990. Ce n'est pas l'objectif de cet article que d'analyser les différences entre ces éditions. Signalons simplement que la dernière publication couvre une période autre que les deux précédentes. 
surmonter sa douleur ${ }^{52}$ : il ingurgite des médicaments à fort pouvoir addictif, mais va essayer de se sevrer, car il veut reprendre le travail :

Aber ich muß davon runter, weil ich versuchen werde, die Arbeit wieder aufzunehmen. Das heißt, die Verlagsleute waren hier. Fritzi [Maxie] ist - so komisch das klingen mag - sowas wie ein Starautor geworden. Das Buch [Guten Morgen] hat hier Aufsehen erregt. Und ich soll einen Band mit Briefen von Fritzi herausgeben ${ }^{53}$.

Même en régime socialiste, l'édition pratique le business as usual. Le livre Guten Morgen Du Schöne ayant connu un succès inespéré, l'éditeur se propose d'exploiter cette manne tant que le public est disposé à s'intéresser à cette auteure et souhaite donc publier les journaux intimes de Maxie. Fred se met immédiatement au travail et dès le 19 décembre il peut annoncer avoir compilé 22 pages du journal ${ }^{54}$ et demande à ses amis de lui prêter des lettres de Maxie afin qu'il puisse les intégrer à l'ouvrage dont la publication est prévue pour 1979. Il pense que Ernst R. en possède environ 300 et les $\mathrm{D}$. une centaine. Dans la préface à l'édition de 1990 de ces cahiers intimes, il tente de justifier sa méthode de sélection, de compilation, de troncage :

Maxie und ich haben oft und lange darüber geredet, wieweit es erlaubt ist, die mündliche Aussage eines anderen zu kürzen und zu verändern, um sie verständlich zu machen. [...] Bei der Arbeit an diesem Buch mußte ich wieder eine Auswahl treffen aus Bergen von beschriebenem Papier. Das war nicht leicht. Ich habe versucht, mich an das zentrale Thema der Aufzeichnungen und Briefe von Maxie $z u$ halten: ihre besessene Suche nach einer neuen Art zu leben, anders zu leben, aufrichtig und schöpferisch zu leben.

La composition des textes des journaux intimes et leur rédaction finale sont donc l'œuvre de Fred et de l'éditrice, qui, elle, dans son exposé à l'instance de censure, signale : "Gesprächspartner für die Arbeit am Manuskript war Fred Wander, Behutsamkeit und Einfühlungsvermögen bestimmten unser Herangehen bei Straffungen und gelegentlichen Kür-

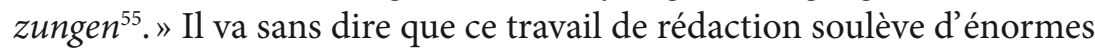
problèmes de censure auxquels vient s'ajouter l'intervention de la censure officielle. En effet, l'un des censeurs propose une liste d'une quin-

52. AFW, lettres du 5 et 7 décembre aux D. et aux R.

53. AFW, 587, lettre à R. du 7 décembre 1977.

54. AFW, 476, lettre du 19 décembre 1977 aux D.

55. AFA, DR 1/2321a, p. 452. Le travail de comparaison entre les cahiers originaux et leur version publiée reste à entreprendre. 
zaine de passages à éliminer, particulièrement les critiques à l'encontre des déformations bureaucratiques en $\mathrm{RDA}^{56}$. Finalement, seules quatre de ces "propositions» seront retenues ${ }^{57}$ - mentionnons en particulier l'élimination du renvoi à Le Pavillon des cancéreux d'Alexandre Soljenitsyne. L'une des censeures ${ }^{58}$ fait un éloge appuyé du manuscrit, même si, elle aussi, propose certaines coupures qui concernent les aventures ou les tentatives d'aventures extra-conjugales de Maxie - concession à la bonne morale officielle ou simple proposition tactique pour détourner l'attention des décideurs ? Ces passages qu'elle avait incriminés ne seront finalement pas rayés de l'ouvrage.

D’ailleurs, la lectrice du Buchverlag der Morgen ne manque pas de signaler une problématique systémique de ce cas de censure, car comment censurer un document autobiographique émanant d'une personne décédée ? Rendre le texte plus conforme à l'idéologie officielle ne reviendrait-il pas à censurer la vie même de l'autobiographe ? : «Mit dem Autor der Dokumente kann nicht mehr verhandelt werden ${ }^{59}$.» Cela ressemblait fort à un coup de poker : c'était tout ou rien, publier ou jeter aux oubliettes. Bien entendu, le terme de censure n'apparaissait nulle part dans ce jeu!

Christa Wolf, qui avait préfacé l'édition en RFA de Guten Morgen et connaissait bien le travail et les projets de son amie, est dubitative quant au bien-fondé et la forme prise par ce projet de Fred de publier des pages du journal et des lettres de Maxie, ainsi que de participer à une version cinématographique de Guten Morgen :

Wir werden also sicher nach der Theater- ${ }^{60}$ auch Kino- und Fernsehfassung ${ }^{61}$ davon kriegen. Das ist ja nicht weiter schlimm, wenn es gut gemacht wird. Nur bei den Tagebuch- und Briefauszügen ist mir mulmig, ich weiß nicht, ob da in ihrem Sinne gehandelt wird, wage nicht mich einzumischen, schelte mich dann dafür als feige... das ganze schmeckt mir zu sehr nach ausnutzen eines „Booms“. Fred sagt, er habe einen "Schock“ und könne nicht mehr in Maxies Tagebücher sehn, nun soll wohl ihre

56. AFA, DR 1/2321a, p. 444-445.

57. Ibid., p. 443.

58. Signalons que cette censeure a encore une belle carrière devant elle : il s'agit de Karin Hirdina qui sera plus tard, à partir de 1989 et la chute du mur, professeure d'esthétique à la Humboldt Universität de Berlin et engagée dans la réforme des structures de celle-ci. Elle décéda en 2009.

59. AFA, DR 1/2321a, p. 451-452.

60. La version scénique de Guten Morgen avait connu un énorme succès en RDA. La première de celle-ci fut mise à l'affiche en 1978 au Deutsches Theater à Berlin.

61. Une version télévisée sous forme de série fut diffusée en 1979-1980. Neuf séquences avaient été préparées, mais les trois premières furent interdites - par la censure. 
Lektorin das Manuskript zu Ende machen... Geht das nicht ein bißchen schnell, wie da die noch atmende Hinterlassenschaft eines Menschen zur Sache gemacht wird, mit der man nach Belieben und Bedarf umspringen kann? Hätten wir sie davor schützen müssen? Hätten wir ein Recht dazu gehabt. Ich weiß einfach nicht ${ }^{62}$.

Christa Wolf, qui avec certitude n'est pas favorable à la censure, semble plaider ici au moins pour une période de réflexion et de prise de distance. Et, certainement n'était-elle pas convaincue que le tandem Fred-lectrice fût la bonne équipe rédactionnelle pour préparer cette publication.

On peut voir, à partir de cet historique de la publication des journaux intimes de Maxie Wander, que le dossier biographique qui sommeille dans ces documents est loin d'être clos, car seule une publication fondée sur une relecture critique de ceux-ci devrait permettre de corriger la lecture proposée par les cahiers «officiels» ou la version fortement fictionnalisée produite par Sabine Zurmühl.

Revenons donc à Sabine Zurmühl ${ }^{63}$ : le projet de biographie a dû être lancé vers la fin du siècle dernier. Aux archives de Berlin se trouvent des lettres qui parlent d'interviews de la journaliste à partir de 2000. Pour Fred Wander, et sa troisième femme Susanne, c'est un stress considérable, Fred s'en ouvre dans une lettre à une amie en France ${ }^{64}$. Lorsque le livre paraît vers Pâques 2001, c'est le choc, Wander est horrifié par la version proposée de la vie de Maxie. Il n’est pas avare de quolibets : «Es ist die größte Gemeinheit, die mir in meinem Leben passiert ist. Ein Buch voller Denunziation, Verleumdung, böser Unterstellungen, Lügen und Trivialitäten $^{65}$.» Il annonce avoir confié le dossier à un avocat berlinois afin que celui-ci obtienne de la justice que certaines affirmations soient interdites de publication. Finalement, l'avocat en question abandonne la procédure craignant une défaite juridique en dernière instance qui eût été ruineuse pour le couple Wander. Ceux-ci changent d'avocat, et la vente de la biographie est effectivement interdite, si l'on en croit l'information communiquée par Fred Wander ${ }^{66}$.

62. Christa Wolf, Briefe 1952-2011, Berlin, Suhrkamp, 2016, p. 357-358, lettre du 25 juillet 1978 à Gerti Tetzner (1936-) qui est une amie commune à Maxie et Christa Wolf. Notaire de profession, elle tenta de vivre de son activité d'écrivain, mais connut de nombreux ennuis avec le régime. Maxie Wander souhaitait que la préface de Guten Morgen fût écrite par «Gerti Tetzner, die das Buch, ganz nach meiner Absicht, vom übrigen FeministinnenRummel absetzen wird». Cité d'après Doreen Mildner, Maxie Wanders „Guten Morgen du Schöne" Protokolle oder Porträts?, mémoire de master, Université de Potsdam 2009, p. 48.

63. Sabine Zurmühl est une ancienne journaliste de la revue féministe Courage.

64. AFW 476, lettre du 16 décembre 2000 à Lisette D.

65. AFW 476, lettre du «mardi après Pâques 2001 », donc probablement du 17 avril.

66. AFW 476, lettre du 24 janvier 2002 à Lisette D. Malheureusement nous ne sommes pas 
Ainsi l'auteur aurait pu, semble-t-il, empêcher la divulgation des informations concernant la collaboration de Wander avec les services de la Stasi dont la tentative de prise d'influence sur les milieux littéraires a été amplement documentée et où Fred Wander occupe une modeste place ${ }^{67}$.

\section{Conclusion}

Fred Wander, par son œuvre et sa biographie, est l'exemple même d'une vie littéraire cherchant à trouver une niche pour ainsi dire «libertaire» dans un système qui en fut le complet contraire, autoritaire par essence, répressif par volonté politique. Grâce à son habileté et à son statut spécial de victime du nazisme et de citoyen autrichien, il sut cependant se faire une place et devenir un auteur à succès dans le champ littéraire de la RDA. Cette position, il dut l'acquérir en contournant les différents écueils de la censure officielle. Sa stratégie première fut donc de ne pas s'ingérer dans les affaires intérieures de ce pays socialiste de langue allemande. Dans un effort d'autocensure et d'autodépassement, il ne se fera remarquer dans aucun des débats entre écrivains et pouvoir politique. Il sut adapter ses écrits à l'horizon d'attente des autorités culturelles, sans cependant faire acte d'allégeance de manière ostentatoire à l'écriture réaliste exigée par le Bitterfelder Weg. Qu'il soit lui-même devenu un acteur-censeur dans la publication de textes de son épouse, Maxie, relève d'une nécessité de protection psychologique intime probablement compréhensible, mais qui demanderait aujourd'hui à être revue. Qu'il ait finalement agi lui-même comme censeur contre la biographie de Zurmühl est plus que regrettable, mais relève de la même problématique psychologique qui cherche à préserver certains secrets intimes, peut-être à tout prix.

En comparant son statut de censuré et de censeur à la contribution de Bourdieu à l'exploration de cette thématique sociologique et littéraire, il semble que Wander montre de manière empirique toute la pertinence des réflexions du sociologue français.

L'écrivain s'en serait probablement bien passé.

parvenu à consulter des documents suffisamment fiables pour confirmer ou infirmer la validité de cette information. Sabine Zurmühl dit n'avoir aucun document (conversation téléphonique avec l'auteur de ces lignes du 5 décembre 2019), que l'éditeur se serait occupé de tout. Ce dernier, qui connut de multiples changements de propriétaire, dit n'avoir aucune information (id., même date.). La censure serait-elle censurée par les censurés ?

67. Joachim Walther, Sicherungsbereich Literatur. Schriftsteller und Staatssicherheit in der Deutschen Demokratischen Republik, Berlin, C. Links, 1996, voir p. 671. 